\title{
WHO's Stop TB Strategy: Progress and Prospects
}

\author{
MUKUND UPLEKAR, DIANA WEIL, and MARIO C. RAVIGLIONE \\ Stop TB Department, World Health Organization, Geneva, Switzerland
}

\begin{abstract}
"In addressing the TB epidemic, including drug-resistant TB and HIV-associated $T B$, the world faces a human rights and health imperative: univenal dccess to early and effective diagnosis, treatment, and cure for TB disease is essential to save lives and prevent transmission. Guided by the vision of the UN MDG 6 $T B$-related targets and by the mandate of strengthening health systems within the context of comprehensive universal primary heat care for all, WHO must work with countries to implement the Stop TB Strateg and the Global Plan to Stop TB."

WHO Strategic and Technical Advisory Growp for Tuberculosis, 2008

\section{Introduction}

Since the passage of the 1991 World Health Assembly (WHA) resolution declaring tuberculosis (TB) as a global public health emergency, the progress in global TB control has been steady (1). In 1994 the internationally recommended TB control strategy, later named DOTS, was launched (2). The DOTS framework was subsequently expanded in 2002 to better address theprevailing constraints to global TB control (3). On World TB Day, March 24, 2006, WNO launched a comprehensive Stop TB Strategy, developed with the engagement of arange of stakeholders and endorsed by the Stop TB Partnership as well as the WHA $(4,5)$. The Stop TB Strategy is built on the foundation of DOTS and underpins the Global Plan to Stop TB, 2006 to 2015 (6,7). Since its launch, the strategy has been adopted and implemented widely by countries including all with a high burden of TB By the end of 2007, nearly 40 million patients had been treated under the DOTS approach. Where DOTS had been provided, treatment success has steadily increased from $77 \%$ in 1994 to $85 \%$ in 2006. TB detection under DOTS increased from an estimated $11 \%$ in 1995 to an estimated $63 \%$ in 2007 . However, in the past two years, the earlier aeceleration has come to an end and the case detection rate is stagnating (8).

This chapter describes the Stop TB Strategy (Table 1) and also summarizes the progress made since its launch. The section below outlines the current challenges to global TB control and the opportunities to address them effectively. Section III presents the goals and the targets of the Stop TB Strategy. The major part of this chapter, section IV, details the six components of the Strategy including recent achievements under each component. On the basis of the recent experience in implementation of the Strategy and to help amplify and clarify the objectives, WHO, in late 2008, has adopted some revision of components and subcomponents of the Stop TB Strategy. Section V presents approaches 
Table 1 The Stop TB Strategy at a Glance

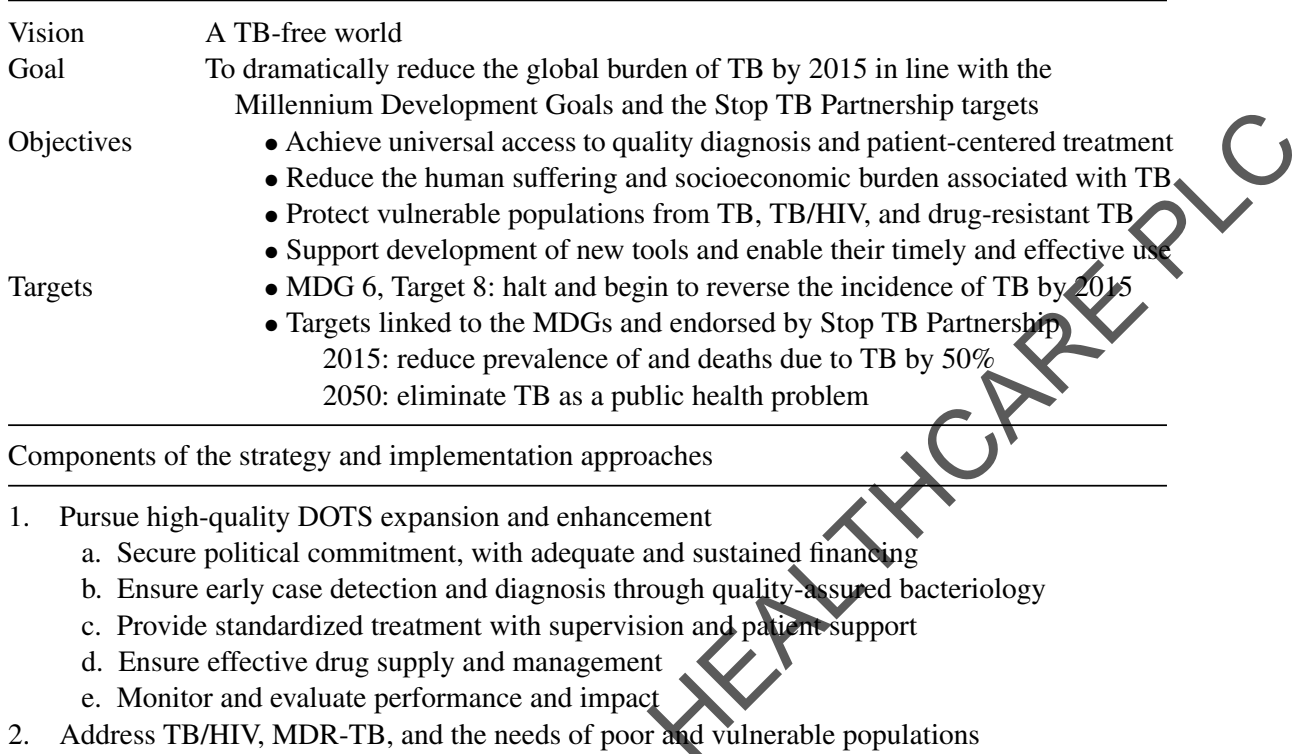

a. Scale up collaborative TB/HIV activities

b. Scale up prevention and management afrmultidrug-resistant TB (MDR-TB)

c. Address the needs of TB contacts and poor and vulnerable populations

3. Contribute to health system strengthening based on primary health care

a. Help improve health policies, human resource development, financing, supplies, service delivery, and information

b. Strengthen infection control in health services, other congregate settings and households

c. Upgrade laboratory netmorks and implement the Practical Approach to Lung Health (PAL)

d. Adapt succes ful approaches from other fields and sectors, and foster action on the social determinants of health

4. Engage all care providers

a. Involve all public, voluntary, corporate, and private providers through Public-Private Mix (PPM) approaches

6. Promote use of the International Standards for Tuberculosis Care

5. Empower people with TB and communities through partnership

a. Pursue advocacy, communication, and social mobilization

b. Foster community participation in TB care, prevention, and health promotion

c. Promote use of the Patients' Charter for Tuberculosis Care

Enable and promote research

a. Conduct program-based operational research

b. Advocate for and participate in research to develop new diagnostics, drugs, and vaccines 
to measuring the progress and the impact of the Stop TB Strategy and related efforts to control TB. The chapter ends with concluding remarks in section VI.

\section{Challenges and Opportunities}

The concerted global efforts to control TB for over a decade have had an important impact on reducing morbidity and mortality. Estimated prevalence and death rates per 100,000 population declined from 296 (1990) to 206 (2007) and from 29 (1990) to 26 (2007), respectively (8). Although TB prevalence and mortality rates are apparently falling, recent trends suggest that the Stop TB Partnership targets of halving 1990 prealence and death rates by 2015 are unlikely to be met globally. In the past two years, the earlier acceleration in detection has come to an end and the case detection rate is now stagnating (9). Particularly, urgent action is needed where the epidemic worsened dramatically in the late 20th century, notably in Africa but also in Eastern Europe. Sub-Saharan Africa has to face the challenge of managing the rapid rise $\mathrm{An}$ TB cases produced by the HIV epidemic, often in places where the human resources and health services are already overburdened. In Eastern Europe, the socioeconomie crisis that followed the dismantling of the Soviet Union in the early 1990s and pelated impoverishment of public health systems contributed to rising incidence and prevalence of TB, including multidrugresistant TB (MDR-TB). Asia also demands serious attention, as it bears two-thirds of the global TB case burden. India, P.R. China and Indonesia rank first, second, and third among countries worldwide in estimated câses. An emerging HIV epidemic and MDR-TB also present important challenges in Asia In all settings, identifying and reaching all those in need of care, especially the poorest among the poor, pose a major challenge. Related to this, efforts to improye TB care and control must progress as an integral part of strengthening primary heatth care (PHC)-based health systems as a whole. At the same time, bringing downincidence and mortality at a much faster pace, fully tackling HIV among TB patients, preventing and treating MDR-TB, and, ultimately, eliminating TB-all depend heayily an the discovery and introduction of new diagnostics, drugs, and vaccines.

WHO and Stop TB partners have developed approaches to overcome some of today's major constraints to TB control. These include focused policy to expand use of newer diaghostic tools and impact measurement (10); public-private mix (PPM) strategies aimed at engaging all care providers, public and nonstate, and partnerships with patients and affected communities in TB care and control $(11,12)$; innovative mechanisms such as the Global Drug Facility (GDF) and the Green Light Committee (GLC) to improve access to quality-assured, affordable drugs for well-designed programs in resource-poor settings $(13,14)$. The collaborative activities that need to be implemented by both TB and HIV/AIDS programs via primary care facilities have been defined (15), and strategies to prevent and manage MDR-TB have been developed and are beginning to be rolled-out (16). New product development partnerships and academic research initiatives have created the first serious pipeline of new diagnostics, drugs, and vaccines to take the world from 19th to mid-20th century technology into the 21 st century-albeit far later than for other urgent concerns such as HIV. Partnerships are being developed across and within countries and among a wide array of stakeholders to respond to health system and 
disease-control challenges. The recent emergence of extensively drug-resistant TB (XDR-TB) has stimulated a new energy in facing TB as a health security threat--an unfortunate wake-up call to the world that moderate advances in facing this age-old disease are not enough (17). Far more bold interventions on insufficient and unsafe public health practice and far more rigorous action in research were needed long before and must be taken up now (18).

Investments in TB have grown dramatically in the last five years, with more rich country attention to development, and the creation of the Global Fund to fight AIDS TB, and Malaria ("The Global Fund") and UNITAID (see below) offer innovation in the sources and modalities of international financing for disease control efforts. Yet, the resource improvements fall far shy of the needs outlined in the Global Plan to Stop TB, 2006 to 2015, especially the interventions required to address TB/HTV and MDR/ XDR-TB (7).

\section{Goals and Targets}

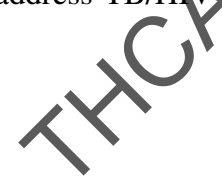

The Stop TB Strategy responds to a vision of a world free of TB. The goal of the Stop TB Strategy is to dramatically reduce the global burden $\triangle \mathrm{TB}$ by 2015 in line with the Millennium Development Goals (MDGs) and the Stop TB Partnership targets, and to achieve major progress in the research and development of new tools needed for TB elimination. The Stop TB Strategy has four major objectives, which are designed to achieve the goal. These are

1. to achieve universal access to high quality diagnosis and patient-centered treatment for all people with $\mathrm{TB}$.

2. to reduce the human suffering and socioeconomic burden associated with TB;

3. to protect vulnerable populations from TB, TB/HIV, and MDR-TB; and

4. to support development of new tools and enable their timely and effective use.

Targets for TB controlhave been established by the WHA (1), by the United Nations as part of the MDGs (19), and by the Stop TB Partnership (7). The Stop TB Strategy is designed to achieye these targets. The WHA targets for 2005 were not met globally by all countries although the Western Pacific region as a whole and 26 countries worldwide did achieve the $7 \%$ case detection and $85 \%$ treatment success rate levels required. The aim beyond 2005 is now to maintain or exceed $85 \%$ treatment success and move toward full care defection. The MDG target relevant to TB (Goal 6, Target 8) is "to have halted and begun to reverse incidence by 2015" (19). The interpretation of Target 8 is that the ipcidence rate of all forms of TB should be falling by 2015. TB indicators have been defined for MDG 6, Target 8 . These are TB prevalence and death rates and the proportion of cases detected and successfully treated under DOTS (Table 2).

The Stop TB Partnership has endorsed two epidemiological targets related to the MDGs: to decrease 1990 TB prevalence and death rates by $50 \%$ by 2015 (7) (Table 2). Achievement of these "impact" targets globally requires sustained progress in implementation, which in turn requires all of the TB care and control innovations already tested and yet more innovation ahead-else the Stop TB Partnership commitment to eliminate TB as a public health problem by 2050 will not be viable. 
Table 2 Millennium Development Goal, Targets, and Indicators for TB and the Stop TB Partnership Targets

\section{Millennium Development Goal 6}

Goal: Combat HIV/AIDS, malaria, and other diseases

Target 8: Have halted by 2015 and begun to reverse the incidence of malaria and other major diseases

Indicator 23: Prevalence and death rates associated with tuberculosis

Indicator 24: Proportion of tuberculosis cases detected and cured under DOTS

Stop TB Partnership Targets

By 2005: At least $70 \%$ of people with infectious TB will be diagnosed (under the DOTS strategy) and at least $85 \%$ cured

By 2015: The global burden of TB (disease prevalence and deaths) will be reduced by $50 \%$ relative to 1990 levels; specifically, this means reducing prevalence to 155 per 190000 per year or lower and deaths to 14 per 100,000 per year or lower by 2015 (including TB cases coinfected with HIV); the number of people dying from TB in 2015 shoud be less than approximately 1 million, including those coinfected with HIV

By 2050: The global incidence of TB disease will be less than 1 case per million population per year

\section{Components of the Stop TB Strategy}

Tackling TB effectively requires addressing at the risk factors that make individuals vulnerable to being infected with TB and to depeloping the disease. It also means reducing the adverse effects of the disease including trs social and economic consequences. Stopping TB must, therefore, be seen within framework of country-owned strategies to reduce poverty, advance development, and strengthen health systems. Therefore, the Stop TB Strategy must be aligned with other strategies and partnerships to address poverty and face all major public health challenges. The main focus of the Stop TB Strategy is on the risk factors that can bedirectly addressed through use of currently available tools for diagnosis, treatment, and prevention of TB as well as the improved tools that are likely to become available in the near future through research and development. The Stop TB Strategy builds on DOTS while also broadening its scope to address remaining constraints and current chalenges in TB control. The six components of the Stop TB Strategy are as follows:

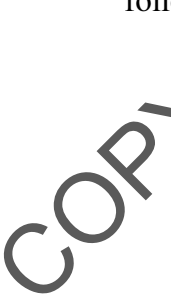

12 Pursue high-quality DOTS expansion and enhancement;

2. Address TB/HIV, MDR-TB, and the needs of poor and vulnerable populations;

3. Contribute to health system strengthening based on PHC;

4. Engage all care providers;

5. Empower people with TB and communities through partnership; and

6. Enable and promote research.

The application of the Strategy has demonstrated that the implementation stage and speed for each of the components of the Strategy will vary depending on the socioeconomic situation, TB epidemiology, the health services landscape, and the soundness of DOTS implementation to date. In promoting the Stop TB Strategy within different country contexts, some observations have been made with regard to its formulation. The first two components provide the technical core of the strategy, while the rest offer approaches 
that will enhance the scope, effectiveness, and impact of the technical components. Also, the first element of the first component--to secure political commitment to TB care and control, with adequate and sustained financing--is essential for the success of the overall strategy not only for basic DOTS efforts.

The paragraphs below describe each component of the Stop TB Strategy with reference to progress in its implementation.

\section{A. Pursue High-Quality DOTS Expansion and Enhancement}

The five elements of DOTS are well established. To address known implementation constraints and challenges, further strengthening of the basic elements of the DOTS approach is required along the following lines.

Secure Political Commitment to TB Care and Control, with Adequate and Sustained Financing

Clear and sustained political commitment by national governments to TB care and control efforts is the backbone of the response in all settings and essential for the whole Stop TB Strategy to be taken forward. The nature of governance and the structure of public health systems notwithstanding, a stewardship function to support TB control efforts through PHC is needed. A national TB program (NTP) with a central unit that establishes norms and policies, supports intermediate and district management, builds capacity, fosters partnerships, solves problems, helps innovation, and provides monitoring and evaluation of services is critical. A medium-term strategicplan for TB control is also needed, with clear process and impact indicators. The Global Plan to Stop TB, 2006-2015, provides a guide for such national plans with benchmarks of progress toward 2015 targets under varying regional scenarios (7). The medium-term plan should be integrated within a national health plan and within poyenty reduction and development frameworks. The plan should address technicaland financial requirements and promote accountability for results at all levels of the health system. Partnerships with the many potential contributors will help improve TB-care in terms of access, equity, and quality. Care can be TB-specific and integrated with broader disease control and other priority public health interventions. Policical commitment should be backed-up by national legislation on TB prevention, care, and control (20). This legislation can lay out the ethical and rights-based principles forprotection from the risk of infection, access to diagnosis and care, responsibility and accountability for actions, and results by health authorities and all service providers. The plan must be supported by adequate and sustained financing with increasing đomestic resources complemented by external funding, where necessary. WHO data collection and analysis of national TB control financing over recent years suggest that there has been substantial recent growth in national financing for TB control in low and middle-income countries as well as in international financing, most notably from the Global Fund. However, new resources are helping to meet more core needs of DOTS implementation, with a greater proportional funding gap in meeting the needs for TB/HIV and MDR-TB investments, as well as advocacy, communications, and social mobilization efforts.

Even with adequate financing, critical deficiencies in the health workforce in the public sector will likely impede progress in many low- and middle-income countries, especially in Africa. Overall, structural and financial changes are required to improve the availability, distribution, and motivation of human resources for health to enable an 
adequate response to all health MDGs (21-24). Changes are needed also to strengthen other health system "building blocks" as noted under the third component of the Stop TB Strategy. A number of global partnerships, including the International Health Partnership and Related Initiatives (IHP+), aim to strengthen coherent national health strategies and harmonize support for these strategies from various sources under compacts among governments, donors, and other partners (25). The Global Health Workforce Alliance is addressing means to overcome some of the major constraints to human resources development, such as reducing the brain drain, reforming civil service payment structures, developing new performance-based incentive structures for geographical and service-level staffing balance, and building preservice and in-service capacity (26).

\section{Ensure Early Case Detection and Diagnosis Through Quality-Assured Bacteriology}

Effective reduction of morbidity, mortality, and transmission of TB depends on accurate and early detection of persons with TB symptoms and their iagnostic screening. The recommended method of TB diagnosis is bacteriology, first using sputum-smear microscopy, and also culture and drug-sensitivity tests when indicated. The latter are crucial for diagnosis and monitoring of smear-negative and drug-resistant TB cases.

Access to quality-assured sputum-smear microseopy means that health services with properly equipped laboratories and well-trainedpersonnel need to be widely available and accessible. This will require additional invesments in the public health laboratory network, including a national TB reference laboratory, in many countries. The laboratory network should be based on the following principles: adoption of national standards in accordance with international guidelines, decentralization of diagnostic services while maintaining high proficiency levels, contimuous interaction between members at various levels of the network, and functiona internal and external quality management including supervision.

Culture and drug-sensitivity testing services should be introduced as quickly as possible as well as periodiesurveys or continuous surveillance of the prevalence of drug resistance. This means that national and subnational reference laboratories need urgent upgrading in mos countries. Referral facilities are also needed to improve diagnosis of sputum smear-negative TB, diagnosis of TB among HIV-positive patients and children, and monitoning of patients with drug-resistant disease. The introduction of new diagnostic tools recommended by WHO, such as culture using liquid media, and line probe assays for rapid genetic identification of drug resistance will be essential for efficient response to diagnostic needs and will take planning and investment to introduce and to scate-up access (27). Maintaining the quality of the laboratory network depends on regular training, supervision and support, and motivation of laboratory staff. Existing public and private laboratories should be used optimally. Generally weak laboratory capacity in face of the need to scale up MDR-TB response poses a great challenge to many NTPs. A Global Laboratory Initiative (GLI) has been established by the WHO and the Stop TB Partnership to strengthen capacity and support mobilization of technical and financial assistance for this challenging process (28). The GLI is already enabling access to new diagnostic tools by working with partners such as the Foundation for Innovative New Diagnostics (FIND), a product development partnership (29), and with UNITAID — an innovative financing mechanism based largely on the generation of financing for HIV, $\mathrm{TB}$, and malaria commodities through airline taxation by selected countries (30). 
Ensuring early and complete case detection requires greater attention for a variety of reasons. Firstly, monitoring of the TB epidemic clearly indicates recent stagnating TB case detection at just above $60 \%$. Ways to increase case detection and sustain it must be identified and implemented rapidly. This translates into the need to progressively shift from detection of TB cases among people presenting symptoms spontaneously to health services toward a more active detection effort. To be productive and cost-effective, such active search for cases should be focused especially on the highly vulnerable populations Secondly, since most of the disease transmission occurs before a TB case is diagnosed and put on treatment, detecting not just more cases but detecting them early enough is important to achieve an impact on the TB epidemic. New approaches should aim at achieving both early and complete case detection. Thirdly, application of new W WO policies on optimization of the number of sputum smears and use of liquideulture could now help in increasing the speed and quality of case detection (27) Enhancing "case detection" will require context-specific approaches for early identification of those with suspected disease, and improving "case diagnosis" will require choosing the diagnostic technique and technology to be employed.

\section{Provide Standardized Treatment with Supervisionand Patient Support}

The mainstay of TB control is organization and administration of standardized treatment countrywide for all adult and pediatric TB. In all cases, WHO's most recent recommendations in published guidelines on patient categorization and management should be followed (31). These guidelines emphasize the use of the most effective, standardized, short-course regimens and of fixed-dose Combinations (FDCs) to facilitate adherence and prevent the risk of acquiring drug resistance. WHO guidelines are also available for management of patients with drug-resistant TB (15).

Supervision and patient support remain the cornerstone of DOTS-based care. Staff responsible for delivery of TB care should identify and address factors that may make patients interrupt or stop treatment. Supervised treatment assists patients in taking their drugs regularly and in completing the treatment, thus helping to achieve cure, prevent development of drug resistance, and, by reducing transmission, protect general public from contracting the disease. Supervision of treatment is meant to ensure adherence on part of both: the providers (in giving proper care and detecting treatment interruption) and the patients (in taking regular treatment). It must be carried out in a context-sensitive and patient friendly manner. Depending on the local conditions, supervision may be undertaken at a health facility, in the workplace, in the community, or at home. A treatment supporter must be identified for each TB patient: a person acceptable to and chosen by the pattent and trained and supervised by health services to guarantee regular intake of drugs

the patient. Patient and peer support groups can help further to promote adherence to eatment.

Locally appropriate measures should be consciously undertaken to identify and address physical, financial, social and cultural, as well as health system barriers, to access TB treatment services. Particular attention should be paid to the poorest and most vulnerable groups. Examples of appropriate actions include providing free diagnosis and treatment, expanding treatment outlets in the poor rural and urban settings, involving providers and treatment supporters in the community that practice and reside close to where patients live, and offering psychological, social, and legal support. Also useful are treatment enablers that can compensate for the indirect costs of care and enable adherence, 
efforts to explicitly address gender issues, improve staff attitudes, enhance communication and social mobilization, and support patient and community groups to help create demand and avail quality-assured support and services. It is essential that these approaches are based on ethical principles where the needs, rights, capabilities, and responsibilities of patients, their families, and their communities are all addressed.

Factors such as the type of drug regimen (daily or intermittent), the type of drug formulation (FDCs or separate drugs), as well as characteristics of the patient should be considered in organizing patient supervision. Use of Directly Observed Treatment (DQT) as a method of supervision recommended by WHO has been a subject of lengthy debates and controlled trials, thanks to the exaggerated attention paid solely to the literal actof watching patients while swallowing pills, rather than viewing it as an integral andessential part of any patient support package designed to guarantee the full cours of treatment and remove any obstacles that may make treatment onerous for patients 32,33 ). Direct supervision of the taking of each dose of drugs is indispensable th the treatment of, for example, patients with psychosocial problems, prison inmates, or patients receiving second-line anti-TB drugs. As TB is a public health problem and its transmission poses a risk to the community, facilitating and ensuring regular intake of all the drugs by the patient for the complete duration is a responsibility of the health care staff and of the national TB program, but it does not mean that healn wotkers must be the supervisors. The whole purpose of undertaking DOT would be lost/f it limits access to care, turns patients away from treatment, or adds to their hardships. The emergence and spread of MDR and XDR-TB further reinforces the absolute necessity of helping a TB patient not to miss any drug doses. Many country TB programs now have considerable experience in identifying adherence promotion stratedies that work or do not work and tailoring treatment supervision to a given ontext TB programs should continue to strengthen patient supervision and suppor with the goal of achieving complete access to a full course of treatment, full treatnentadherence, and patient satisfaction with care.

\section{Ensure Effective Orig Supply and Management}

An uninterrupted and sustalned supply of quality-assured anti-TB drugs are fundamental to TB control. For this purpose, an effective drug procurement, distribution, and management system is essential and should be provided through the essential drug-supply system if it exists, as part of PHC. Anti-TB drugs should be available free of charge to all TB patients, both because many patients are poor and TB treatment has benefits that extend to socien as a whole-successful treatment prevents disease transmission. Legislation related fo drug regulation should be in place and use of anti-TB drugs by all providers should be strictly monitored. The use of FDCs of proven bioavailability and innovative packaging, such as blister packs and patient kits, can help improve drug supply logistics as well as drug administration, reduce nonadherence to treatment, and prevent development of drug resistance. The GDF and the GLC meant for access to second-line anti-TB drugs offer support to secure financing and procurement of quality-assured anti-TB drugs at competitive prices and also facilitate access to strengthen their drug management capacity $(13,14)$. The GDF is governed by WHO on behalf of the Stop TB Partnership and utilizes an independent procurement agent which directly procures first-line anti-TB drugs with GDF resources or those of the recipient country or a third party, a donor agency for example (13). The GLC is managed by WHO and vets proposals for MDR-TB case management and then enables access to concessionally priced second-line drugs 
and technical assistance for implementation (14). The establishment of UNITAID has been a major step in resolving gaps in financing for anti-TB drugs, especially pediatric formulations and second-line drugs for MDR-TB treatment (30).

Since the launch of the Stop TB Strategy, availability of quality-assured and affordable anti-TB drugs has improved. For example, the prequalification process for pediatric formulations of FDCs has been accelerated via mechanisms including pooled procurement by the GDF, the involvement of UNITAID, and provision of technical assistance from the WHO prequalification project. Despite progress, drug shortages continue to occur in all regions at both central and peripheral levels. Better monitoring and management of drug supply and distribution are still required (9). Improving supply systems is among the major stated concerns for health system strengthening efforts in general.

Monitor and Evaluate Performance and Impact

Establishing a reliable system to monitor and evaluate TB performance and impact is vital. This system needs to be embedded within overall national health information systems, and it depends on regular communication between the central and peripheral levels. The system must encompass standardized recording of individual patient data, including information on case detection by category for quarterly case notification and on treatment outcomes, which are then used to compile quarterly treatment oucomes in cohorts of patients. These data, when compiled and analyzed, can be used at the faélity level to monitor treatment outcomes, at the facility and the district level to identify local problems as they arise, and at a provincial or national level to ensure support and secure consistency of TB control across geographical areas. Lastly, data are used nationally and internationally to evaluate the performance of each country and forepldemiological surveillance. Regular program supervision should be carried out o verify the quality of information and to address performance problems.

Increasingly, countries have additional information at their disposal, including results of sputum culture, results of drug sensitivity tests, and HIV test results. WHO has recently developed and shared with countries a revised framework for TB recording and reporting (34). This system incorporates recording of additional data that should be routinely collected to enable monitoring of the implementation of the Stop TB Strategy. Countries haya also begun to revise their recording and reporting systems to reflect the various gomponents and subcomponents of the Stop TB Strategy. Many countries are beginning to shift to electronic and internet-based recording and reporting systems, which is assisting with increased speed of data collection and review and depth of analysis.

1 To make the best use of data at all levels, many countries will need to do more to train staffin the analysis and interpretation of data as well as in the use of computer software that can greatly facilitate this work. As electronic recording systems become more widely available, consideration should be given to storing individual patient data, which will make it possible to not only carry out more detailed analyses using aggregated data but also raise the need for increased care in maintaining confidentiality of information.

In view of the need for each country to assess the progress of its control efforts toward achieving established national or global targets, a number of approaches to impact measurement have been recommended (35). While the definitive tool would be to monitor incidence trends through routine well-functioning surveillance systems, disease prevalence surveys and death registration systems (or verbal autopsy where those are not in place) are also useful methods to assess the burden. Each country should plan impact 
measurement, which is of increasing importance in designing national programs, in mobilizing and justifying financial resources for program operations and in reviewing and revising strategies.

\section{B. Address TB/HIV, MDR-TB, and the Needs of Poor and Vulnerable Populations}

\section{Scale Up Collaborative TB/HIV Activities}

By suppressing the immune response, HIV promotes the progress of recent and latent infection due to Mycobacterium tuberculosis to active TB. It also increases the rate of recurrent TB. The HIV epidemic has caused a substantial increase in the proportion of TB cases that have smear-negative pulmonary and extrapulmonary TB $\mathrm{TH}$-positive smear-negative pulmonary TB patients have inferior treatment outcomes and higher early mortality compared with HIV-positive smear-positive pulmonary TB patients. In the long term, only effective control of the HIV epidemic will reverse the associated increase in TB incidence. However, until then, interventions to reduce HIV-related TB morbidity and mortality need to be implemented (35).

WHO has published a policy document on collaborative TB/HIV activities (15). This is a twelve-point policy package incorporating thee, broad categories: establishing the mechanisms for collaboration, decreasing the burden of TB in people living with HIV/AIDS, and decreasing the burden of HIV TB patients. The second category is focused on efforts that can be taken uRpincipally under the guidance of HIV programs and partners through primary gare facilities and is referred to as the three I's: intensified case finding, isoniazid preventive therapy, and infection control. Special attention has been given to the scale-up of these interventions in Africa and to high-level commitment by governments and partners to support the full TB/HIV policy package (36). A Ministerial Forum op the theme was organized at the UN in June 2008, under the leadership of the UN Special Knvoy to Stop TB (37). UNAIDS, PEPFAR, and the Global Fund, among others, are expanding their efforts on TB/HIV work. In several African countries, including Malawl, Rwanda, and Kenya, remarkable progress has been made in expanding acces to WIV testing for TB patients, reaching more than three-fourths of their patients (9). This trend is being repeated in other countries now and the challenge ahead is ensuring easy access to care for those testing positive for HIV and those HIV-infected persons testing positive for TB infection and other diseases. Far more work still needs to be one to enable universal access to antiretroviral therapy for qualifying TB patients and to pursue all of the three I's.

\section{Scale Up Prevention and Management of MDR-TB}

Global surveillance of anti-TB drug resistance indicates that it is present everywhere and that it is especially prevalent in countries of the former Soviet Union and some provinces of P.R. China. The highest ever reported levels of MDR-TB disease (up to 20\% of all new patients in some former Soviet countries) were published in the 2008 WHO Report on Anti-Tuberculosis Drug Resistance in the World (17). This report also documented the rising number of countries (more than 50 by late 2008) with reported cases of XDR-TB, a more lethal form of MDR-TB that is more difficult and more costly to treat. Evidence from Southern Africa suggests that where XDR-TB is introduced into congregate settings, like health centers and hospitals with significant numbers of immunocompromised individuals 
such as those infected with HIV, the consequences can be severe with extremely high mortality. Once introduced, XDR-TB can stretch low-income country capacity to provide adequate public health protection and patient care (38).

In the areas where MDR-TB has reached alarming levels, TB cannot be controlled if MDR-TB prevention and management are not explicitly addressed, alongside strong basic DOTS services. This means that special attention is given to infection control, early screening and diagnosis for drug-resistant $\mathrm{TB}$, and adequate and appropriate treatment of MDR-TB. Detection and treatment of all forms of drug-resistant TB should eventually be an integral part of NTP activities. Although this may be challenging, experience shows that it strengthens the program's overall capacity to implement TB control measures. The key actions to prevent and control drug-resistant TB include a comprehensive care framework including models of care for different levels of the health system, patient support, a reliable supply of diagnostics and drugs, and measures to improve the guidance and regulation of MDR-TB treatment by all health care providers, including hospitals, clinics, private practitioners, and community-based treatment supporters.

Management of MDR-TB under programmatic conditions is feasible, effective, and cost-effective when implemented in the context of a well-functioning TB control program and based on WHO's policy guidelines on the programmatic management of MDR-TB (16).

The GLC reviews project and programmatic proposals for the initiation and scale-up of programmatic management of MDR TB and enables access to secondline drugs for approved efforts (14). GLC-approved projects have now been initiated in 60 countries. However, in the 27 countries estimated by WHO to have the heaviest burdens of MDR-TB, few have national plans devised for full national scale-up of MDR-TB response. In the next few years, it ill be imperative for all to be working urgently in building the support and capacity needed to mount such responses.

\section{Address the Needs of TB Contacts, and of Poor and}

Vulnerable Populations

TB programs need to pay specific attention through focused approaches and activities to TB contacts and to all poor and vulnerable populations who face a higher risk of contracting TB, barriers to accessing care, and problems in adhering to treatment. Guidance on identifying and managing contacts of TB patients, at home and outside, needs to be provided to all countries. Contact tracing results in early case detection and treatment as wellas provision of preventive therapy where appropriate. There may be significant challenges in establishing contact tracing and follow up mechanism in health systems with limited human resources. The well-established but widely underutilized intervention of contact tracing deserves systematic implementation by NTPs in high-burden ountries (HBCs).

Populations that are more vulnerable to contract TB include the very poor; women and children; malnourished persons; smokers, alcoholics, and injecting drug users; persons with comorbidities such as diabetes; prison populations; internally displaced persons and refugees; migrating workers and others working in ill-ventilated and congested workplaces; indigenous people; and specific ethnic groups and other marginalized populations. Special situations requiring extra attention for the design of TB prevention and treatment efforts include unexpected population movements such as political unrest, war, natural disaster, and other conditions causing refugee movements. In these circumstances, there 
may be a disruption of social networks. In all these contexts, there is also the imperative to help ensure that ethics and human rights tenets are supported, as the populations and communities of concern may also be those that lack voice and other means to ensure that their rights are upheld and advanced.

TB services need to adapt to address the specific needs of high-risk groups and to support TB prevention and control in special situations (39-43). NTPs must not lose the sight of the fact that TB is primarily a disease of poverty and it is often the poorest and the most vulnerable who have problems in availing services meant for them. Ministries of Health should ensure that TB diagnosis and drugs are free of charge, that the density of services near the poorest populations is adequate, and that the local communities are effectively engaged to make care and health promotion more accessible to the poor.

In the face of stagnating TB case detection in spite of wide coverage and implementation of basic DOTS services, focused action is needed to reach the most vulnerable through targeted strategies and to ensure that NTPs collaborate with al partners working to meet other health and development needs of these populations.

\section{Contribute to Health System Strengthening Based on PHC}

Strengthening health systems is now seen as an urgent prerequisite for achieving all the health MDGs. There is a recognition that disease control responses have helped build commitments in the health sector and toward system strengthening, but the demands are great. There are new partnerships and hightevel cooperation to help build momentum for the scale-up of health interventions while also improving the human resources and supportive systems that enable delivery af heatth services and outcomes. WHO has called for a renewal of commitment to PH principles as a guiding framework in improving systems and outcomes (44). This is why coherent contributions by national TB programs to these processes are important and will benefit the program as well as the whole system. The section below presents the core elements of this component of the Stop TB Strategy.

Help Improve Health Policies, Human Resource Development, Financing, Supplies, Service Delivery, and Information

Health system strengthening is defined as "improving capacity in some critical components of heatth systems, in order to achieve more equitable and sustained improvement across health services and outcomes." TB control programs and their partners should participate actively in country-led and global efforts to improve the "building blocks" of systems. Which include leadership and governance, the health workforce, health financing, medical products and technologies, service delivery, and information (45). Health sy stem strengthening also means working across all levels of systems and with all actors in the public sector, nonstate sector, civil society, and communities. This includes efforts to improve sector strategies and plans, align all efforts with these plans, increase coherent implementation of the plans, monitor performance to achieve health outcomes, and help devise, test, and share new ways of working. The top concerns today include increasing the predictability and sustainability of health financing; implementing key means to retain, expand, and improve the capabilities of the health workforce; and reinforce PHC to support universal health coverage and the MDGs. WHO guidelines produced in 2002 to help orient and align TB control efforts within health system reforms are still highly 
relevant today (46), and a new guide to how programs can contribute has been developed (47). NTPs and partners should help to reduce any duplication or distortions caused in local systems by the rapid scaling up and/or expanded financing for TB efforts and help to build coordination across disease-specific initiatives.

\section{Strengthen Infection Control in Health Services, Other Congregate Settings and Households}

The emergence and spread of drug-resistant TB has provided a pointed reminder to address infection control measures seriously and systematically. There have been a number of welldocumented outbreaks of TB, including MDR and XDR-TB, that have occurred in health care facilities (48-51). There is also good evidence that incidence of TB in congregate settings and households exceeds the incidence among the general population.Particularly, the high frequency of HIV infection in many health care facilities and the cancern of the spread of drug resistant TB make infection control an issue of paramount importance.

TB infection control (TB-IC) is a combination of controlmeasures: (a) managerial activities (e.g., planning), (b) administrative controls (e.g., separation of patients), (c) environmental controls (e.g., ventilation), and (d) use of personal protective equipment (e.g., respirators). All are aimed at minimizing the ris $\mathrm{k}$ of TB transmission within populations. It is critical that each control measure is implemented in a patient-centered approach. Evidence shows that implementation of these measures reduces transmission of TB in health care facilities (52). This in turn cecreases transmission of TB among patients, health workers, and visitors, and thereby can avert TB cases and deaths. All facilities, public and private, caring for TBparients and for persons suspected of having TB should implement the TB-IC measures VB-IC should complement general infection control efforts and those targeting other airborne infections.

To date, only very few countries bave strategies to implement TB-IC interventions. TB-IC strategies require coordinated efforts of multiple stakeholders not only within ministries of health, justice infrastructure, etc., but also through engagement with civil society and technical partners. The multisectoral nature of interventions makes TB-IC a challenge and addressing it requires both political commitment and coordination at higher levels across relevant ministries. The best combination of controls will be informed by local programmatie climatic, and socioeconomic conditions.

The updated WHO policy on TB-IC provides guidance on what to do and how to prioritize TB IC at national level. While the focus of the recommendations is on health racilities, the document also provides guidance on preventing TB transmission in congregate settings and households (53). TB prevention, diagnosis, and care objectives. Both areas of work aim to improve core clinical and public health practice through innovation in the efficient use of the health workforce, infrastructure, and technologies.

The GLI (28), noted above, provides one launch pad to support national public health laboratory networks by, among other roles, providing norms, standards, and 
practical guidance for the use of new diagnostic tools and facilitating technical assistance coordination and support for development of system-wide laboratory strengthening plans as part of sector strategies. Today, disease-specific initiatives may offer one of the best avenues for financing laboratory service delivery and improvements. Improving TB laboratory capacity will address major barriers in overall laboratory systems, including infrastructure, supply chain management, and human resource development. Fundamental to this work, therefore, is NTP collaboration with and support to public health laboratory leadership and staff.

PAL is among the innovations initiated within the TB control community that can strengthen the health system as a whole. Pulmonary TB often manifests as a cough, and persons with TB symptoms first present themselves to primary care services as andefined respiratory patients (54). By linking TB control activities to proper management of all common respiratory conditions, TB programs and staff implementing DOTS services at local level can help to improve the efficiency and quality with which careis provided. PAL aims at improving the quality of the management of respiratory patients in PHC settings. It is a patient-centered approach to diagnosis and treatment of common respiratory illnesses encountered in PHC. It promotes a symptom-based and integrated management system and seeks to standardize service delivery through the development and implementation of clinical guidelines. PAL is intended to ensure the coordination among different levels of health care and between TB control programs and general health services. Implementing PAL can improve TB case detection and also enhance the quality of care for common respiratory illnesses (55).

PAL has been initiated in more than 35 countries and is being scaled up within the PHC network in approximately 10 countries. However, technical capacities to support countries to implement PAL activities need to be developed at global and regional levels. Countries where PAL is at the expansion phase need to generate data on routine basis to show the impact of this approach on the integration of respiratory care in PHC, the management of TB suspects. TB detection, and drug consumption.

\section{Adapt Successful Approaches from Other Fields and Sectors, and Foster Action on the Social Determinants of Health}

To respond to all six elements of the Stop TB Strategy, TB programs and their partners can adapt approaches that have been applied in other priority public health fields and even in other sectors, and build further on some of the common systems that are already in place This may include, for example, further integration of TB-control activities within the community and PHC outreach pursued in maternal and child health programs, social mobilization along the lines used by HIV/AIDS programs and partners, regulatory actions that have been used in tobacco control, innovations for human resources development offered by the wider education field, logistics innovations offered in a range of other fields, or financing initiatives and methods to reach the poorest that have been set up for financing vaccine development and immunization services. It can also include further collaboration with broader information platforms (household surveys, etc.) to advance TB surveillance and program monitoring. Effective integration of delivery systems depends on testing, adapting, scaling up, and evaluating common approaches.

There are a range of social determinants of TB exposure, disease and treatment outcomes. There is increasing documentation of the nature and scope of some of these TB-related determinants, but far more needs to be done to stimulate "upstream" and 
"downstream" actions to eliminate these risk factors and/or reduce their impact (56). The WHO and partners are now acting to more explicitly and urgently address social determinants and resulting equity concerns, through promoting "health for all policies" across sectors. NTPs must be aware of the global and local evidence, help contribute to improving the knowledge base, and act to influence change. Some actions can be taken by NTPs themselves through their own monitoring and evaluation, policy and programmatic guidance, and related capacity building of the health workforce, patient and communities. Many of these steps can and should be taken in collaboration with others

\section{Engage All Care Providers}

\section{Involve All Public, Voluntary, Corporate, and Private Providers}

Through PPM Approaches

In most settings, patients with symptoms suggestive of TB seek care troma wide array of health care providers within and outside the public sector TB sernces. These may include private clinics operated by formal and informal practitioners andinstitutions owned by the public, private, voluntary, and corporate sectors (e.g., general and specialty public hospitals; nongovernmental organizations (NGOs); faith-based organizations (FBOs); prison, military, and railway health services; and heaith/insurance organizations). These non-NTP providers may serve a large proportion of TB symptomatic patients while not always applying recommended TB management practices or reporting their cases to NTPs. Some settings have large private and NGO/FBQ sectors, while others have public sector providers (such as general and specialty hospitals) that operate outside the structure of NTPs. Evidence suggests that failure toinvokve all care providers used by TB symptomatic patients hampers case detection, delays diagnosis, causes improper diagnosis as well as inappropriate and incomplete treatment, increases drug resistance, and places a large and unnecessary financial burden on patients (57-59).

WHO has produced guidelines on how to engage all care providers in TB control (11). The feasibility, effectiveness, and cost-effectiveness of involving different types of care providers using PPMapproach have been demonstrated $(60,61)$. NTPs should aim to engage all care providers in DOTS implementation to help achieve the TB control targets, improve access to care, standardize the quality of TB care across providers, and save costs of care for patients. Priority should be given to identifying and setting up collaboration with health care providers who diagnose and treat a large number of TB patients and suspects and are used by the poor sections of the population. The major limiting factors in working with individual private providers have been their large number and weak rganization. An efficient way of engaging with private practitioners would be through rofessional associations. NTPs should engage with professional associations to involve individual providers in TB control. Where they are weak, it would be worthwhile to help develop capacity of such organizations to contribute effectively to TB care and control.

\section{Promote Use of the International Standards for TB Care}

The International Standards for Tuberculosis Care (ISTC) have been formulated based on a wide global consensus of appropriate practices in TB diagnosis and treatment $(62,63)$. They should be actively promoted and used to help engage all care providers in DOTS implementation and are particularly complementary to the PPM approaches described above. The standards of care are evidence based. They can be used to secure a broad 
base of support for TB control efforts from NTPs and professional, medical, and nursing societies, academic institutions, NGOs/FBOs, and HIV-focused organizations. They can also help to create peer pressure to encourage providers to conform to the principles and serve as a basis for preservice and in-service training $(62,63)$. Early experience in using the ISTC as an advocacy and technical tool to foster collaboration between NTPs and professional associations has been encouraging.

Making involvement of all care providers an essential component of the Stop TB Strategy has stimulated countries to embark on initiating and scaling up locally appropriate strategies to systematically engage diverse providers operating outside the scope of NTPs. All HBCs have explicit policies to help implement PPM programs; many countries have begun scaling up and over a dozen of them have funds from the Global Fund for this purpose. In 2007, for example, private providers in Pakistan contributed about a quarter of all smear-positive cases notified in the country. In the Philippines, $\pi$ thareas where PPM activities are being implemented (and where one-third of the country's population lives), approximately $13 \%$ of TB cases were reported through/PPM units operated by diverse for-profit, voluntary, educational, and corporate institutions. PPM approaches are also being extended to TB/HIV and MDR-TB implementation. Weak capacities in both public and private sector remain a major constraint to scale up PPM. To help address this partly, for the first time in October 2008, representatives of national professional associations from $22 \mathrm{HBCs}$ joined NTP managers to diseuss collaborative ways to make rapid progress with wide application of ISTC.

\section{E. Empower People with TB, and Communities Through Partnership}

One of the greatest gains in global healhin the last decade has been the active creation of new partnerships among a wide range of stakeholders. Many partnerships and resulting collaborative initiatives and fonancing mechanisms are mentioned in several sections of this paper. One area where partnership has particular significance and resonance is in the area of empowering those affected by disease and communities, particularly the most vulnerable. The process of empowerment requires strong actions taken by the target groups themselver as well as supportive efforts and solidarity provided by NTPs and other partners.

\section{Pursue Advocacy, Communication, and Social Mobilization}

In the context of wide-ranging partnerships for TB control, advocacy, communication, and social mobilization can help build greater commitment to, and effectiveness in, fighting TB. Advocacy is intended to secure support of key constituencies in relevant local, national, and international policy discussions and is expected to prompt greater aecountability from governmental and international actors. Communication is concerned with informing and enhancing knowledge among the general public and people with TB and empowering them to express their needs and take action. Encouraging providers, at the same time, to be more receptive to expressed concerns and views of people with TB and community members will make TB services more responsive to actual needs. Social mobilization is the process of bringing together all feasible and practical intersectoral allies to raise people's knowledge of, and demand for, quality TB care and health care in general to assist in the delivery of resources and services and to strengthen community participation for sustainability (64). Advocacy, communication, and social mobilization 
efforts in TB control should be linked with overarching efforts to promote public health and social development.

\section{Foster Community Participation in TB Care, Prevention and Health Promotion}

Community participation in TB care, prevention, and health promotion implies establishing a working partnership between the health sector and the community, especially poor and vulnerable populations in general and TB patients, current as well as cured, in particular. Enabling people with TB and communities to be informed about TB, to ephance general awareness about the disease and to share responsibility for their health can leadto effective patient empowerment and community participation, by increasing he demand for health services and bringing care closer to the community. For this purpose, TB programs should provide support to frontline health workers and involyecommunities to help create an empowering environment by, for instance, facilitating setting up of patient groups, encouraging peer education and support, and linking with othercommunity-level partnerships and local initiatives. Programs and health workers should intervene under the notion of subsidiarity, where they aim to provide support that complements and supplements that offered by the community and does not aim toduplicate or supplant action where the community already has capacity. Community volunteers also need regular support, motivation, instruction, and supervision. Evidence shows that community-based TB care is cost-effective compared to hospital-based care and other ambulatory care models $(65,66)$. Community involvement in planning, implementation, monitoring, and evaluation is essential to sustain communit TB initiatives. WHO has recently published evidence-based guidelines to help countries scale up community TB care and prevention. The overall principles outlined here arepresented in depth in that publication (12).

\section{Promote Use of the Patients' Charter for TB Care}

Developed by patients from around the world, the Patients' Charter outlines the rights and responsibilities of peepla with TB and complements the International Standards for Tuberculosis Care intended for health care providers (67). It is based on the principles of various internationa and national charters and conventions on health and human rights. It also addresses some of the underlying ethical principles that are critical in public health, primary care practice, and research. It aims to empower people with TB and the communities and make the patient-provider relationship mutually beneficial. The charter sets out the ways in which patients, communities, health care providers, and governments can work as partners and help enhance the effectiveness of health services in general and TB care in particular. The charter provides a useful tool to guide action to achieve greater inyolvement of people in TB care. There is urgent work to be done to support efforts in countries to adopt and respond to the principles, given varying degrees of development and organization of their health systems.

\section{F. Enable and Promote Research}

\section{Conduct Program-Based Operational Research}

The Stop TB Strategy consolidates DOTS implementation and involves the implementation of several new approaches to tackle challenges facing NTPs and those affected by TB. To put them into practice, program-based operational research should be a core 
component of NTP work. Designing and conducting locally relevant operational research can help identify problems, determine workable solutions, test them in the field, and plan for scale up. For this purpose, collaboration between program managers and researchers is essential. Acquiring basic skills in identifying and addressing issues related to program operations and performance can help program managers to initiate operational research in collaboration with researchers and academia. This can then help to sustain and strengthen TB control efforts by expanding existing activities and introducing new strategies effectively. For this purpose, sustainable partnerships and networks need to be established productive collaboration on operational research. Also joint projects should be considered within wider networks of those working on "research for health" (68).

\section{Advocate for and Participate in Research to Develop New Diagnostics,} Drugs, and Vaccines

Existing tools for prevention and treatment of TB make standard TB care demanding for both patients and their care providers. The tools include a gentary-old, tedious, and weakly sensitive smear microscopy test for diagnosis (although it is the best method available today to identify highly infectious cases), and a relatiyely long "short-course" chemotherapy with several drugs. A truly effective vaccine is lacking. The need to rely on these tools has substantially hindered the pace of progress in global TB control. Increasing advocacy and voiced demand from countries $/ n$ most need of improved tools is critical to help enable financing and conduct more basic research and development for TB prevention, care, and control. The Stop TB Partnership's Working Groups on New Diagnostics, Drugs, and Vaccines are helpong increased understanding of the needs and growth of the pipelines in all fields throughinnovative public-private product development partnerships. TB programs should actively encourage and participate in this process. In addition to advocating for research and capacity building for research in developing nations, NTPs and their national research counterparts can help enable and speed up the field testing of new products and prepare for swift adoption and roll out of new tools as they become available.

The Stop TB Partnership sponsors a subgroup on "Retooling," which signifies the process of planning for guidelines and tools revision, capacity building, and stakeholder engagement needed to successfully and rapidly adopt new tools into national TB control efforts. The sabgroup involves a wide range of stakeholders such as program staff, civil society, researchers, product developers, research and program donors, and technical assistange providers. The subgroup is helping by providing guidance tools to help foster this process (69). One example is that checklists are available to help ease the planning

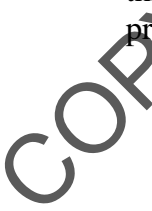
ocess for introducing liquid culture methods at country level (70).

\section{Measuring Global Progress and Impact}

\section{A. Measurement of Program Outcomes and Impact on Burden of Disease}

The Stop TB Strategy is designed to achieve the MDG and related Stop TB Partnership targets (explained in section "Goals and Targets") as well as to stimulate regular monitoring to assess the progess toward targets. Table 3 shows the indicators that apply for each of the targets and how they can be measured. 
Table 3 Selected Indicators for Monitoring TB Programs

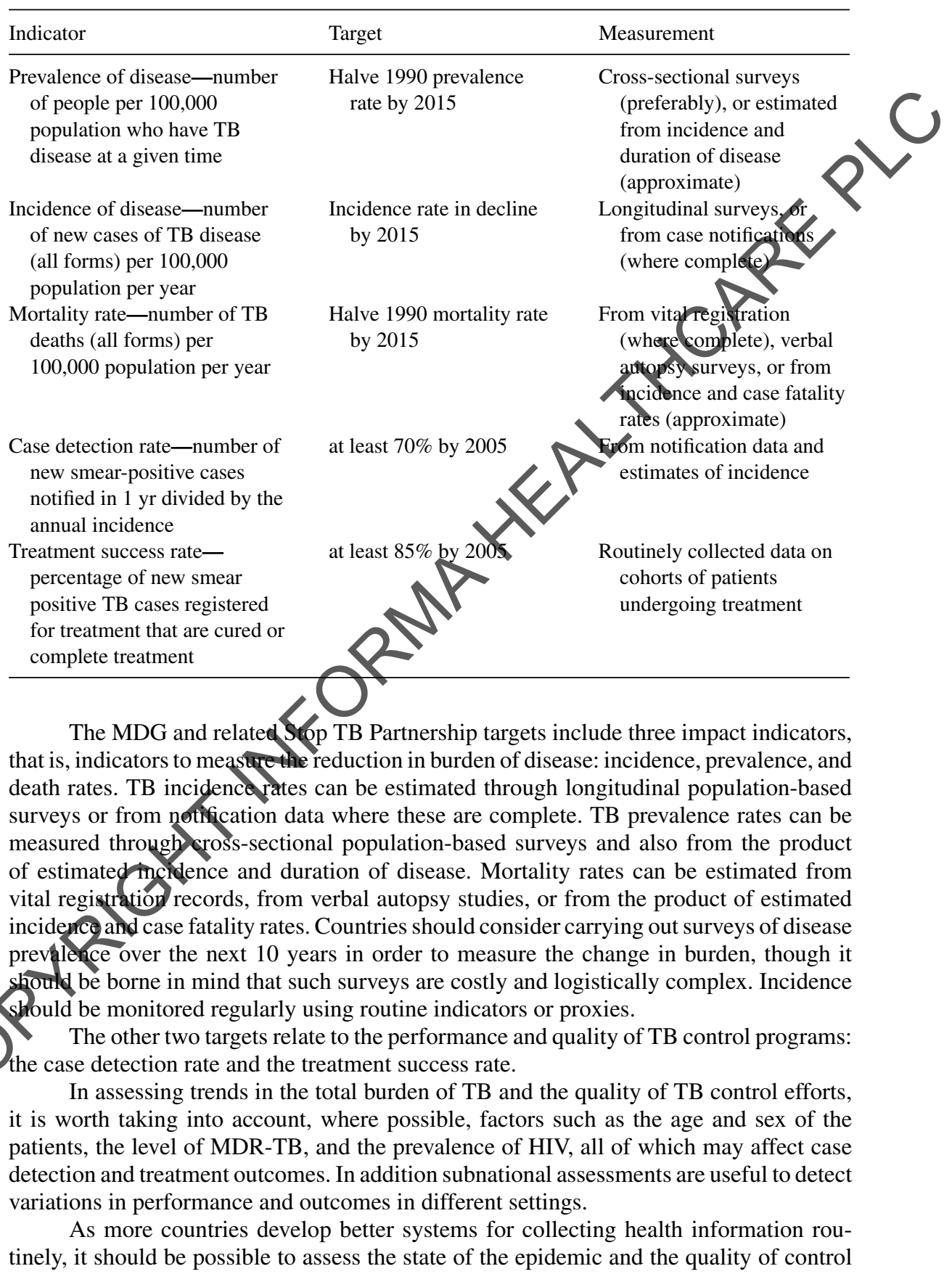


using annual TB surveillance data, together with data from vital registration. To complement and check the quality of routine surveillance data, it will be important to carry out population-based surveys of disease prevalence or infection. The WHO Task Force on TB impact measurement has recommended a set of epidemiological criteria to guide selection of countries that should undertake prevalence of disease surveys during the period up to 2015 (35). Of the 57 countries that meet the criteria, 30 have plans to carry out a national or subnational survey.

\section{B. Financing for TB Control}

Achieving the MDG and Stop TB Partnership targets will require increased, predictable, and sustained financing for TB control, as reflected in Component 1 of the Stop TB Strategy. Financing of TB control needs to be monitored and evaluated at subnational, national, and international level to document trends in NTP budgets, available funding for these budgets, funding gaps, expenditures, and total TB control costs (total TB control costs include costs reflected in NTP budgets plus costs associated withusing general health services staff and infrastructure). A consistent categorization of budget line items and funding sources should be used to allow analysis of changes aver time. These categories may be modified periodically, for example, to refled the introduction of a major new source of funding or when a major shift in strategy alters the line items for which it is relevant to collect data. WHO collects financial data through a questionnaire, which is sent to all countries annually. These data areanalyzed and presented in the annual WHO report on global TB control.

Some of the key indicators that are rdevant to financial monitoring and evaluation of TB control include the annual NTPbudget requirement, the NTP budget per patient treated, the percentage of the NTPbudget that is funded, the percentage of the NTP budget that is funded by the government (including loans), the percentage of available funding that is spent, the total annual cost of TB control, the cost per patient treated, and the cost per patient successfully treated. Unike the outcome and impact indicators described above, national and international targets for financing have not been established. Nevertheless, monitoring changes over time is useful and should be undertaken regularly. WHO has recently prepared a planning and budgeting tool to help countries develop costed plans in line with the global targets (71). Workshops conducted subsequently have supported over 50 countries including $15 \mathrm{HBCs}$ to use the tool for plan development. These costed TB plans can then be embedded with national health strategies and national budgets. In times of financial crisis, when reductions in financing and increased vulnerability can have serious public health consequences, it is particularly critical to have well-costed disease control plans, along with strong monitoring mechanisms of program efficiency, outcomes, and impact.

\section{Conclusion}

The Stop TB Strategy comprehensively addresses the problem of TB. It is equipped with the lessons from DOTS implementation, field-tested approaches to tackle current challenges, early experiences of implementing the components beyond DOTS, renewed efforts in developing new tools, and a strong Stop TB Partnership of all stakeholders. The Strategy also provides the basis and the context for the Global Plan to Stop TB, 
2006-2015 (7). This inclusive plan exploits the various synergies and new approaches and carries an estimated cost of US \$67 billion over 2006 to 2015, US \$56 billion for implementation and the rest for research. The strength of global efforts to control TB lies in the coordinated and collaborative efforts of the Stop TB Partnership. With a clear global strategy and related global plan, the framework is in place for unprecedented efforts in TB control over the next six years. Experience to date shows that adopting all components of the Strategy takes significant coordination and stewardship capacity of National TB Programs and full implementation will require substantial resources. Although funding is rising, more is needed if we are to achieve the MDG and the Stop TB Partnership fargets for TB control and set ourselves on the path toward elimination of this ancient scourge of humanity.

\section{References}

1. World Health Organization. Forty-Fourth World Health Assenbly Geneva, Switzerland: WHO, 1991. WHA44/1991/REC/1.

2. World Health Organization. Framework for Effective Tuberculosis Control. Geneva, Switzerland: WHO, 1994. WHO/TB/94.179.

3. World Health Organization. An expanded DOTS Framavork for Effective Tuberculosis Control. Geneva, Switzerland: WHO, 2002. WHO/CDS/TB/2002.297.

4. Raviglione MC, Uplekar MW. WHO's new Stop TB Strategy. Lancet 2006; 367: 952-955.

5. World Health Organization. Sixtieth World Health Assembly. Geneva, Switzerland: WHO, 2007. WHA60/2007. Available at:http//mww.who.int/gb/ebwha/pdf_files/WHASSA_ WHA60-Rec1/E/reso-60-en.pdf. Accessed Ianuary 14, 2009.

6. Dye C, Maher D, Weil D, et al. Targets forglobal tuberculosis control. Int J Tuberc Lung Dis 2006; 10(4):460-462.

7. Stop TB Partnership and WHO. Global Plan to Stop TB 2006-2015. Actions for LifeTowards a World Free of/Tuberdulosis. Geneva, Switzerland: WHO, 2006. WHO/HTM/ $\mathrm{STB} / 2006.35$.

8. World Health Organization, Global Tuberculosis Control-Surveillance, Planning, Financing, WHO Report 2009. Geneva, Switzerland: WHO. 2009.

9. World Health Organization. Global Tuberculosis Control-Surveillance, Planning, Financing, WHO Report2008. Geneva, Switzerland: WHO, 2008. WHO/HTM/TB/2008.393.

10. World Health Organization. Report of the Eighth Meeting of the Strategic and Technical Advis ry Group for Tuberculosis (STAG-TB). Geneva, Switzerland: WHO, 2008.

11. World Health Organization. Guidance on Implementing Public-Private Mix for DOTS. Engag10 AllHealth Care Providers To Improve Access, Equity and Quality of TB Care. Geneva, Switzerland: WHO, 2006. WHO/HTM/TB/2006.360.

World Health Organization. Community Involvement in Tuberculosis Care and Prevention. Towards Partnerships for Health. Guiding Principles and Recommendations Based on a WHO Review. Geneva, Switzerland: WHO, 2008. WHO/HTM/TB/2008.397.

13. World Health Organization. Ten Million Treatments in Six Years-GDF Achievements Report. Geneva, Switzerland: WHO, 2007. WHO/HTM/STB/2007.40.

14. World Health Organization. Report of the Annual Meeting of the Green Light Committee (GLC) of the Working Group on MDR-TB of the Stop TB Partnership. Geneva, Switzerland: WHO, 2008. WHO/HTM/TB/2008.409.

15. World Health Organization. Interim Policy on Collaborative TB/HIV Activities. Geneva, Switzerland: WHO, 2004. WHO/HTM/TB/2004.330.

16. World Health Organization. Guidelines for the Programmatic Management of Drug-Resistant Tuberculosis. Geneva, Switzerland: WHO, 2008. WHO/HTM/TB/2008.402. 
17. World Health Organization. WHO/IUATLD Global Project on Anti-tuberculosis Drug Resistance Surveillance. Anti-tuberculosis Drug Resistance in the world. Geneva, Switzerland: WHO, 2008. Report no. 4, WHO/HTM/TB/2008.394.

18. Raviglione MC. Facing extensively drug-resistant tuberculosis-a hope and a challenge. N Engl J Med 2008; 359(6):638-638.

19. United Nations Statistics Division. Millennium indicators database. Available at: http:// unstats.un.org/unsd/mi/mi_goals.asp. Accessed January 13, 2009.

20. World Health Organization. Good Practice in Legislation and Regulations for TB Controf. An Indicator of Political Will. Geneva, Switzerland: WHO, 2001. WHO/CDS/TB/2001.290.

21. World Health Organization. Human Resources Development for TB Control: Repory of a Consultation Held on 27-28 August 2003. Geneva, Switzerland: WHO, 2004. WHOHTM/ TB/2004.340.

22. World Health Organization. Check-list for the Review of Human Resource Development Component of National Plans to Control Tuberculosis. Geneva, Switzerfand WHO, 2005. WHO/HTM/TB/2005.354.

23. World Health Organization. The World Health Report 2006: Working Together for Health. Geneva, Switzerland: WHO, 2006.

24. World Health Organization. Planning the Development of Human Resources for Health for implementation of the stop TB strategy—A Handbook. Geneva Switzerland: WHO, 2008. WHO/HTM/TB/2008.407.

25. International Health Partnership and Related Initiatiyes 2007, IHP+. Available at: http://www.internationalhealthpartnership.net. Accessed December 18, 2008.

26. Global Health Workforce Alliance. World Health Organization, 2009. Available at: http://www.who.int/workforcealliance/en/. Accessed January 14, 2009.

27. World Health Organization. New Techpologies for Tuberculosis Control: A Framework for Their Adoption, Introduction and Implementation. Geneva, Switzerland: WHO, 2007. WHO/HTM/STB/2007.40.

28. Global Laboratory Initiative (GL World Health Organization. Available At: http://www. who.int/tb/dots/laboratory/gl/en/index.html. Accessed January 12, 2009.

29. Foundation for Innovative New- Diagnostics (FIND). Delivering on the promise: Five years of progress towards more effective diagnostic tests for poverty-related diseases. Available at: http://www.finddiagnostics.org/news/resources/delivering_on_the_promise_sep08.pdf. Accessed February 15, 2009.

30. UNITAID. Inovative Financing for health the air tax-A journey to access. December 2008. Available At: http://www.unitaid.eu/images/stories/unitaiden.pdf. Accessed February 15,2009

31. Worl Health Organization. Implementing the Stop TB Strategy: A handbook for National Tuberculosis Control Programmes. Geneva, Switzerland: WHO, 2008. WHO/HTM/ TB12008.401.

32. Volmink J, Garner P. Directly observed therapy for treating tuberculosis. Cochrane Database Syst Rev 2003; 1:CD003343.

33. Volmink J, Matchaba P, Garner P. Directly observed therapy and treatment adherence. Lancet 2000; 355(9212):1345-1350.

34. Norval PY, Heldal E, L'Herminez R, et al. Revising the tuberculosis recording and reporting information system. Int J Tuberc Lung Dis 2008; 12(3)(suppl 1):17-19.

35. Dye C, Bassili A, Bierrenbach AL, et al. Measuring tuberculosis burden, trends, and the impact of control programmes. Lancet Infect Dis 2008; 8(4):233-243. Epub 2008 Jan 16.

36. Nunn P, Williams B, Floyd K, et al. Tuberculosis control in the era of HIV. Nat Rev Immunol $2005 ; 5: 819-826$.

37. Stop TB Partnership. First HIV/TB Global Leaders' Forum held at UN Headquarters. STOP TB News. July 2008. Available at: http://www.stoptb.org/resource_center/assets/documents/ STBNEWSJULY08.pdf. Accessed January 13, 2009. 
38. Andrews JR, Shah NS, Gandhi N, et al. Multidrug-resistant and extensively drug-resistant tuberculosis: Implications for the HIV epidemic and antiretroviral therapy rollout in South Africa. J Infect Dis 2007; 196(suppl 3):S482-S490.

39. World Health Organization. Addressing Poverty in Tuberculosis Control: Options for National TB Control Programmes. Geneva, Switzerland: WHO, 2005. WHO/HTM/TB/2005.352.

40. World Health Organization. Tuberculosis Care and Control in Refugee and Displaced Populations: An Interagency Field Manual, 2nd ed. Geneva, Switzerland: WHO, 2007. WHO/HTM/TB/2007.377; WHO/CDS/DCE /2007.2.

41. Coninx R. Tuberculosis control in complex emergencies. Bull World Health Organ 2007 85:637-640.

42. Gayer M, Connolly MA. Tuberculosis control in refugee and displaced populations An: Raviglione MC, ed. Reichman and Hershfield's Tuberculosis: A Comprehensive, International Approach, 3rd ed. Part B. New York: Informa Healthcare USA, Inc., 2006:907-919.

43. World Health Organization. Tuberculosis Control in Prisons: A Maneral for Programme Managers. Geneva, Switzerland: WHO, 2000. WHO/CDS/TB/2000.281)

44. World Health Organization. The World Health Report 2008: Primary Health Care Now More Than Ever. Geneva, Switzerland: WHO, 2008.

45. World Health Organization. Everybody's Business-Strengthening Health Systems To Improve Health Outcomes. WHO's Framework for Action. Geneva, Switzerland: WHO, 2007.

46. World Health Organization. Contributing to Health System Strengthening: Guiding Principles for National Tuberculosis Programmes. Geneva, Swrkerland: WHO, 2008. WHO/HTM/ $\mathrm{TB} / 2008.400$.

47. World Health Organization. Expanding DOTS in the Context of a Changing Health System. Geneva, Switzerland: WHO, 2003. WHQKCDS/TB/2003.318.

48. World Health Organization. Tuberculosis Infection Control in the Era of Expanding HIV Care and Treatment. Addendum o WHO Guidelines for the Prevention of Tuberculosis in Health Care Facilities in Resource-Limited Settings, 1999. Geneva, Switzerland: WHO, 2006.

49. Frieden TR, Sterling T, Pablos-Mendez A. The emergence of drug-resistant tuberculosis in New York City. N Engl MMed. 1993; 328(8):521-526.

50. Moro ML, Gori A, Errante 1 , et al. An outbreak of multi drug-resistant tuberculosis involving HIV-infected patients of two hospitals in Milan, Italy. Italian Multidrug-Resistant Tuberculosis Outbreak Study Group. AIDS 1998; 12(9):1095-1102.

51. Gandhi NR, Moll A, Sturm AW, et al. Extensively drug-resistant tuberculosis as a cause of death inpatients co-infected with tuberculosis and HIV in a rural area of South Africa. Lancet 2006; 368: $575-1580$.

52. Harries AD, Hargreaves NJ, Gausi F, et al. Preventing tuberculosis among health workers in Matawi. Bull World Health Organ 2002; 80:526-531.

53. World Health Organization. WHO policy on TB infection control in health-care facilities, eongregate settings and households. Geneva, Switzerland: WHO, 2009. WHO/HTM/TB/ 2009.419

World Health Organization. Practical Approach to Lung Health (PAL). Manual on Initiating PAL Implementation. Geneva, Switzerland: WHO, 2008. WHO/HTM/TB/2008.410.

55. Ottmani S, Mahjour J. The practical approach to lung health strategy for integrated respiratory care. In: Raviglione MC, ed. Reichman and Hershfield's Tuberculosis: A Comprehensive International Approach, 3rd ed. Part B. New York: Informa Healthcare USA, Inc., 2006: 1059-1081.

56. World Health Organization. Commission on social determinants of health. Social determinants of tuberculosis. In: Blas E, ed. Social Determinants of Health and Public Health Programmes. Report of the Priority Public Health Conditions Knowledge Network. Geneva, Switzerland: WHO, 2008. 
57. Lönnroth K, Tin-Aung, Win-Maung,et al. Social franchising of TB care through private general practitioners in Myanmar-an assessment of access, quality of care, equity, and financial protection. Health Policy Plan 2007; 22:156-166.

58. Uplekar M. Involving private health care providers in delivery of TB care: Global strategy. Tuberculosis 2003; 83:156-164.

59. Lönnroth K, Uplekar M, Arora VK, et al. Public-private mix for DOTS implementation: What makes it work? Bull World Health Organ 2004; 82(2):580-586.

60. Pantoja A, Lönnroth K, Lal SS, et al. Economic evaluation of public-private mix for TB, India. Part I Int J Tuberc Lung Dis 2009, 13(6):695-704.

61. Pantoja A, Floyd K, Unnikrishnan KP, et al. Economic evaluation of public-private mix for TB, India. Part II Int J Tuberc Lung Dis 2009, 13(6):705-712.

62. Hopewell PC, Pai M. Tuberculosis, vulnerability and access to quality care. AMA 2005; 293:2790-2793.

63. Hopewell PC, Pai M, Maher D, et al. International standards for tubepeulosis care. Lancet Infect Dis 2006; 6:710-725.

64. World Health Organization, Stop TB Partnership. Advocacy, Communication and Social Mobilization (ACSM) for Tuberculosis Control: A Handbook for Country Programmes. Geneva, Switzerland: WHO, 2007.

65. Okello D, Floyd K, Adatu F, et al. Cost and cost-effectiveness community-based care for tuberculosis patients in rural Uganda. Int J Tuberc Lung Dif 2003; 7(9)(suppl 1):S72-S79.

66. World Health Organization. Community Contriburion to TB Care: Practice and policy. Geneva, Switzerland: WHO, 2002. WHO/CDS/TB/2002.318.

67. Patient's charter for Tuberculosis care. World Care Council, 2006. Available at: http://www. imaxi.org/pdf/PatientsCharterEN2006.pdf. Accessed January 13, 2009.

68. The Lancet. The Bamako call to action: Research for health. Lancet 2008; 372(9653): 1855.

69. World Health Organization, Stop TB Partnership. Engaging Stakeholders for Retooling TB Control. Geneva, Switzerland: WHO, 2008.

70. Stop TB Partnership. Checklist of key actions for the use of liquid media for culture and drug susceptibility testing (DST). Retopling Task Force - February 2008. http://www.stoptb.org/ retooling/assets/documents/Retooling\%20Task\%20Force\%20-\%20Liquid\%20CultureDST\%20Checklist\%20-820Feb2008.pdf. Accessed January 13, 2009.

71. Floyd K, Pantoja A. Financial resources required for tuberculosis control to achieve global targets set for 2015. Bull World Health Organ. 2008; 86(7):568-576. 